\title{
Continuous metabolic syndrome score (siMS) enables quantification of severity of cardiometabolic affliction in individuals not presenting with metabolic syndrome
}

\author{
Sebekova $\mathrm{K}^{1}$, Sebek $\mathrm{J}^{2}$ \\ Institute of Molecular BioMedicine, Faculty of Medicine, Comenius University, Bratislava, Slovakia. \\ kata.sebekova@gmail.com
}

\begin{abstract}
BACKGROUND: Assessment of degree of cardiometabolic affliction in subjects not presenting with metabolic syndrome (MS) yet, would be helpful in the management of preventive health maintenance.

OBJECTIVES: To evaluate continuous metabolic syndrome score (siMS) in estimation of severity of cardiometabolic affliction in individuals not presenting with MS.

METHODS: We analyzed data from 3166 volunteers (56 \% females) aged $\geq 16$ years. siMS score was calculated as waist/height/0.5 + fasting plasma glucose (FPG)/5.6 + triacylglycerols (TAG)/1.7 + systolic blood pressure (SBP)/130 - high-density lipoprotein cholesterol (HDL-C)/1.02 (males) or 1.28 (females). In siMS quintiles, numbers of individuals presenting with 0-to- $5 \mathrm{MS}$ components were calculated. MS was considered as the presence of any 3 out of its 5 components.

RESULTS: $33 \%$ of participants without MS scored $\geq 4$ th quintile; $13 \%$ of those free from MS components; 49 $\%$ of participants presenting with 1 , and $83 \%$ of those displaying 2 MS components. $11 \%$ of individuals presented with MS, all but 1 displayed siMS within the 2 upper quintiles.

CONCLUSIONS: Considerable proportion of individuals without MS presented with siMS in range displayed by individuals presenting with MS. SiMS might be useful in estimation of severity of cardiometabolic affliction prior to manifestation of MS, to identify individuals requiring early intervention to counteract developing pathological processes (Tab. 1, Ref. 21). Text in PDF www.elis.sk.

KEY WORDS: continuous metabolic syndrome score, metabolic syndrome components.
\end{abstract}

\section{Introduction}

Metabolic syndrome (MS) is a cluster of abnormalities that, if occurring together, impose an increased risk of development of chronic degenerative diseases, such as type 2 diabetes and cardiovascular diseases, even in young individuals $(1,2)$. Current diagnosis of MS is based on dichotomous classification, requiring either presence of any three out of the five criteria (e.g., central obesity, elevated blood pressure, elevated triacylglycerols (TAG), low high density lipoprotein cholesterol (HDL-C), and elevated fasting plasma glucose (FPG) (3); or presence of central obesity and any 2 out of remaining above mentioned criteria (4).

${ }^{1}$ Institute of Molecular BioMedicine, Faculty of Medicine, Comenius University, Bratislava, Slovakia, and ${ }^{2}$ Institute of Materials \& Machine Mechanics, Slovak Academy of Sciences, Bratislava, Slovakia

Address for correspondence: K. Sebekova, MD, DrSc, Institute of Molecular BioMedicine, Medical Faculty, Comenius University, Sasinkova 4, SK-811 08 Bratislava, Slovakia.

Phone: +421.2.59357429, Fax: +421.2.59357631

Acknowledgement: The study was partially funded by The Scientific Grant Agency of the Ministry of Education, Science, Research and Sport of the Slovak Republic and the Slovak Academy of Sciences (APVV), grant No. 1/0637/13; and The Slovak Research and Development Agency (VEGA), grant No. 0447-12.
Dichotomous classification enables a simple, and unequivocal diagnosis of MS, but it does not allow for assessment of continuously rising cardiometabolic affliction. To this point, different approaches to calculate continuous MS score have been proposed (5-7). Continuous MS scores are precise, provide a way of tracking the changes in severity of cardiometabolic burden over time, and to evaluate its severity in individuals not presenting with MS yet. However, these scores are sample (e.g. sex- ethnicity/race-, and age) specific, not allowing between-population comparison, and often are not easy to calculate (5-7). The recently proposed siMS score $(8,9)$ is easy to calculate and comparable across different studies and populations, since it normalizes the individual measures to the accepted international standards $(3,4,10)$. Its utility in following-up individual patients has been documented (8). As the continuous MS score is particularly suitable for detecting an increased cardiometabolic risk prior to the manifestation of MS (5-8), we asked what proportion of individuals not presenting with MS will score within the range of siMS score generally displayed by patients with MS. We assumed that the prevalence of these subjects might be rather high, as Slovaks present the highest cardiovascular mortality among the European Union countries (11). To confirm our hypothesis, we retrospectively calculated the siMS score in individuals formerly participating as volunteers in cross-sectional clinical studies and expressed frequency of sub- 


\section{5-678}

jects manifesting zero to five MS components in each siMS score quintile.

\section{Participants and methods}

We analyzed data from individuals participating during the period 2009-2012 in studies focusing on the prevalence of MS and its components. All volunteers were White Caucasians of Central European descent, residing, working or studying in western Slovakia. They were recruited via advertisements, or information provided at work or at schools. Exclusion criteria were any acute or chronic disease, particularly treatment for hypertension, dyslipidemia, diabetes, and participation in weight reducing programs, in females also pregnancy and lactation. From the database of 3750 volunteers aged 12-to- 81 years, we extracted data on individuals aged $\geq 16$ years, in whom data on body weight, height, waist circumference, blood pressure, FPG, TAG and HDL$\mathrm{C}$ concentrations were available. Limiting the age to $\geq 16$ years enabled to employ the same MS classification criteria in the whole cohort. Full-aged participants signed a written consent to participate, those aged $<18$ years gave a verbal asset, and a written consent from their caregivers was obtained. The studies were approved by local Ethics Boards and conducted in accordance with the Declaration of Helsinki.

Anthropometric measurements were performed by qualified and trained medical personnel, according to the same protocol. Briefly, height was measured using stadiometer, body weight using electronic scales, waist circumference using flexible tape. Body mass index (BMI) and waist-to-height ratio were calculated. Blood pressure was measured in a sitting position after 10 minutes rest using a sphygmomanometer. The mean of last 2 measurements out of 3 was recorded.

Blood was sampled after overnight fasting from antecubital vein. FPG, TAG, and HDL-C were analyzed using standard laboratory methods.

siMS score was calculated according to Soldatovic et al (8), as follows:

siMS score $=$ Waist $/$ Height $/ 0.5+$ FPG/5.6 + TAG/1.7 + $\mathrm{SBP} / 130$ - HDL-C/1.02 (males) or 1.28 (females)

Waist and height were measured in cm; FPG, TAG, and HDL in $\mathrm{mmol} / \mathrm{l}$, and SBP in $\mathrm{mm} \mathrm{Hg}$. Quintiles of siMS score were calculated.
MS components were classified as present, if $\mathrm{SBP} \geq 130 \mathrm{~mm}$ $\mathrm{Hg}$ and/or DBP $\geq 85 \mathrm{~mm} \mathrm{Hg}$, if TAG $\geq 1.7 \mathrm{mmol} / \mathrm{l}$ ), HDL-C; < $1.03 \mathrm{mmol} / 1$ in males and $<1.29 \mathrm{mmol} / 1$ in females, or $\mathrm{FPG} \geq$ $5.6 \mathrm{mmol} / \mathrm{l}$. Participants presenting waist-to-height ratio $\geq 0.5$ were classified as centrally obese (10). In each siMS score quintile, we expressed the number and frequencies of individuals free from MS components, and those presenting with 1,2, 3, 4 or 5 components.

Data were presented as the mean \pm standard deviation, median and interquartile range (IQR), or as counts and percentages. Frequencies were compared using Chi-square test, $\mathrm{p}<0.05$ was considered significant. Data were analyzed using the SPSS v.16 for Windows software (SPSS, USA).

\section{Results}

The study group consisted of 3166 individuals ( $56 \%$ women). The mean age was $22.8 \pm 11.0$ years, BMI averaged $23.6 \pm 4.6 \mathrm{~kg}$ / $\mathrm{m}^{2}$, waist-to-height ratio averaged $0.46 \pm 0.07$; SBP averaged $118 \pm$ $15 \mathrm{~mm} \mathrm{Hg}$, DBP averaged $74 \pm 9 \mathrm{~mm} \mathrm{Hg}$, FPG averaged $4.9 \pm 0.5$ $\mathrm{mmol} / \mathrm{l}$, TAG concentration averaged $1.03 \pm 0.64 \mathrm{mmol} / \mathrm{l}$, and that of HDL-C concentration $1.38 \pm 0.33 \mathrm{mmol} / 1.23 \%$ of participants presented with central obesity, $25 \%$ had elevated blood pressure, $8 \%$ presented elevated FPG, $11 \%$ showed elevated TAG, and 23 $\%$ low HDL-C concentrations. siMS score ranged between 0.65 to 7.08, with median of 2.09 and IQR 1.79-2.48.

Approximately $50 \%$ of participants did not manifest any sign of MS, $28 \%$ presented with one component, $11 \%$ presented with two components, $6 \%$ manifested three components, $4 \%$ displayed four components, and in $1 \%$ of participants all five components of MS were present (Tab. 1).

Among participants manifesting MS ( $\mathrm{n}=349)$, all but one presented with siMS score within the 4 th and 5 th quintile (Tab. 1). Thus, $11 \%$ of our participants, who presented with MS accounted for $27 \%$ of those with siMS in upper 2 quintiles. Remaining 73 $\%$ of participants with siMS score in upper 2 quintiles did not fulfil 3 MS criteria, and $16 \%$ did not even present with a single MS component.

About $13 \%$ of participants free from any MS component presented with siMS score within the 4th and 5th quintile (Tab. 1). Forty-nine percent of individuals manifesting one component of MS displayed siMS score in the range of the upper two quintiles

Tab. 1. Distribution of individuals presenting with zero to five components of metabolic syndrome according to siMS score quintile.

\begin{tabular}{|c|c|c|c|c|c|c|}
\hline \multirow[b]{2}{*}{ No. of MS components } & \multicolumn{5}{|c|}{ siMS score quintiles } & \multirow[b]{2}{*}{ All } \\
\hline & $\begin{array}{c}1 \mathrm{Q} \\
(0.65-1.72) \\
\mathrm{n}=633\end{array}$ & $\begin{array}{c}2 \mathrm{Q} \\
(1.721-1.98) \\
\mathrm{n}=633\end{array}$ & $\begin{array}{c}3 \mathrm{Q} \\
(1.981-2.23) \\
\mathrm{n}=634\end{array}$ & $\begin{array}{c}4 Q \\
(2.231-2.62) \\
n=633\end{array}$ & $\begin{array}{c}5 \mathrm{Q} \\
(2.621-7.08) \\
n=633\end{array}$ & \\
\hline 0 (n; (\% of subjects not presenting with MS components)) & $580(36.4 \%)$ & $469(29.4 \%)$ & $340(21.3 \%)$ & $189(11.9 \%)$ & $16(1.0 \%)$ & $1594(100 \%)$ \\
\hline $1(\mathrm{n} ;(\%$ of subjects presenting with 1 MS component $))$ & $47(5.3 \%)$ & $153(17.3 \%)$ & $253(28.7 \%)$ & $319(36.1 \%)$ & $111(12.6 \%)$ & $883(100 \%)$ \\
\hline 2 (n; (\% of subjects presenting with 2 MS components)) & $6(1.7 \%)$ & $11(3.2 \%)$ & $40(11.8 \%)$ & $108(31.8 \%)$ & $175(51.5 \%)$ & $340(100 \%)$ \\
\hline 4 (n; (\% of subjects presenting with 4 MS components)) & 0 & 0 & 0 & 0 & $131(100 \%)$ & $131(100 \%)$ \\
\hline 5 (n; (\% of subjects presenting with 5 MS components)) & 0 & 0 & 0 & 0 & $38(100 \%)$ & $38(100 \%)$ \\
\hline
\end{tabular}

$\mathrm{Q}$ - quintile; No. - number; MS - metabolic syndrome 
(Tab. 1). Eighty-three percent $(n=730)$ of participants presenting with one component of MS were lean, $17 \%(n=153)$ presented with central obesity. Proportion of individuals displaying siMS score within the two upper quintiles was similar in lean participants presenting with one MS component, and individuals manifesting only central obesity ( $48 \%$ and $51 \%$, respectively, $\mathrm{p}_{\text {chi }}=0.534$ ). Among individuals displaying two components of MS, $83 \%$ had siMS score within the 4 th and 5 th quintile. Thirty-two percent (n = 109) of participants presenting with two MS components did not display central obesity, and among them $83 \%(n=90)$ displayed siMS score within two upper quintiles. Among centrally obese individuals presenting with an additional MS component $(\mathrm{n}=231), 193(84 \%)$ showed siMS score $\geq 4$ th quintile $\left(\mathrm{p}_{\mathrm{ch}} \mathrm{vs}\right.$. centrally lean $=0.821$ ).

\section{Discussion}

MS score describes the continuum of the processes underlying abnormalities in the components of MS. It reflects how individual components of MS cluster together, and the current severity of cardiometabolic affliction, even in subjects not presenting with MS. Similarly to our data calculated using siMS score, specific population-derived continuous MS score rises with the number of manifested components of MS $(12,13)$. Moreover, population-derived MS scores show a significant relationship with different markers of cardiometabolic risk, such as those of inflammation and fibrinolysis, adipokines, adhesion molecules (14) or subclinical atherosclerosis $(12,15)$. Unlike e.g. the Framingham Risk Equation (16) or the AUSDRISK score (17), continuous MS severity scores were not specifically formulated to be risk predictors. However, recent studies document that they are significantly associated with long-term risk of development of coronary heart disease (18), myocardial infarction (19), type 2 diabetes $(19,20)$, as well as of cardiovascular and overall mortality $(19,21)$.

The presence of MS according to dichotomous classification indicates an increased risk to develop cardiometabolic events. In our analysis, except for one individual, those presenting with MS displayed siMS scores in the range of 2 upper quintiles, indicating an above average cardiometabolic burden. Similarly scored about 33 out of 100 participants not presenting with MS, and even about 13 out of 100 not presenting with any component of MS. This prevalence is quite high, when we consider that we analyzed subjects participating as volunteers in clinical studies, e.g. individuals, who are likely to be interested in their health status. Thus, additional studies on a representative sample of the population are needed to clarify the real prevalence. To our knowledge, similar data from other populations are not available. Between-countries comparison employing siMS score would be of interest, as cardiovascular mortality in Slovaks is the highest in the European Union (11). Since we analyzed siMS scores retrospectively, we neither could follow-up potential changes in individual siMS scores over time, nor assess siMS score as an outcome predictor. However, simplicity of siMS calculation and its availability in Excel spreadsheet or Android application $(8,9)$ allows the physician for tracking of changes in severity of cardiometabolic affliction in individual patients over time.

In conclusion, in clinical practice, calculation of siMS might help to estimate the severity of cardiometabolic affliction in so called "low-risk" subjects, e.g. individuals, who do not present with MS components yet. Proportion of these individuals is not negligible, and they logically escape the attention of the physician, and thus the opportunity of being early motivated to implement changes in lifestyle, which could slow-down or even interrupt pathological processes. Availability of automated calculation of siMS score using the electronic health record could facilitate its wider use toward identification of patients with severe cardiometabolic affliction.

\section{References}

1. Ford ES. Risks for All-Cause Mortality, Cardiovascular Disease, and Diabetes Associated With the Metabolic Syndrome. Diabetes Care 2005; 28 (7): $1769-1778$.

2. Expert Panel on Integrated Guidelines for Cardiovascular Health and Risk Reduction in Children and Adolescents: Summary Report 2011; 128 (Suppl. 5): S213-S256.

3. Alberti KG, Eckel RH, Grundy SM et al. Harmonizing the Metabolic Syndrome A Joint Interim Statement of the International Diabetes Federation Task Force on Epidemiology and Prevention; National Heart, Lung, and Blood Institute; American Heart Association; World Heart Federation; International Atherosclerosis Society; and International Association for the Study of Obesity. Circulation 2009; 120 (16): 1640-1645.

4. Zimmet $\mathbf{P}$, George $\mathbf{K}$, Alberti MM et al. The metabolic syndrome in children and adolescents - an IDF consensus report. Pediatr Diabetes 2007; 8 (5): 299-306.

5. Wijndaele K, Beunen G, Duvigneaud N et al. A continuous metabolic syndrome risk score - Utility for epidemiological analyses. Diabetes Care 2006; 29 (10): 2329.

6. Eisenmann JC. On the use of a continuous metabolic syndrome score in pediatric research. Cardiovasc Diabetol 2008; 7: 17.

7. DeBoer MD, Gurka MJ. Clinical utility of metabolic syndrome severity scores: considerations for practitioners. Diabetes Metab Syndr Obes 2017; 10: 65-72.

8. Soldatovic I, Vukovic R, Culafic D, Gajic M, Dimitrijevic-Sreckovic V. siMS Score: Simple Method for Quantifying Metabolic Syndrome. Plos One 2016; 11 (1): e0146143.

9. Vukovic R, Milenkovic T, Stojan G et al. Pediatric siMS score: A new, simple and accurate continuous metabolic syndrome score for everyday use in pediatrics 2017; 12 (12): e0189232.

10. Ashwell M, Gibson S. A proposal for a primary screening tool: 'Keep your waist circumference to less than half your height'. BMC Med 2014; 12: 207.

11. Mortality from cardiovascular diseases. Health at a Glance 2015: OECD Indicators. Paris: OECD Publishing; 2015.

12. Pandit D, Chiplonkar S, Khadilkar A, Kinare A, Khadilkar V. Efficacy of a continuous metabolic syndrome score in Indian children for detecting subclinical atherosclerotic risk. Int J Obes (Lond) 2011; 35 (10): 1318-1324. 


\section{5-678}

13. Heshmat R, Heidari M, Ejtahed HS et al. Validity of a continuous metabolic syndrome score as an index for modeling metabolic syndrome in children and adolescents: the CASPIAN-V study. Diabetol Metab Syndr 2017; 9: 89.

14. Olza J, Aguilera CM, Gil-Campos M et al. A Continuous Metabolic Syndrome Score Is Associated with Specific Biomarkers of Inflammation and CVD Risk in Prepubertal Children. Ann Nutr Metab 2015; 66 (2-3): 72-79.

15. Prochotska K, Kovacs L, Vitariusova E, Feber J. Is arterial stiffness predicted by continuous metabolic syndrome score in obese children? J Am Soc Hypertens 2016; 10 (1): 47-54.

16. D’Agostino RB, Vasan RS, Pencina MJ et al. General cardiovascular risk profile for use in primary care - The Framingham Heart Study. Circulation 2008; 117 (6): 743-753.

17. Chen L, Magliano DJ, Balkau B et al. AUSDRISK: an Australian Type 2 Diabetes Risk Assessment Tool based on demographic, lifestyle and simple anthropometric measures. Med J Aust 2010; 192 (4): 197-202.
18. DeBoer MD, Gurka MJ, Golden SH et al. Independent associations between metabolic syndrome severity \& future coronary heart disease by sex and race. J Am Coll Cardiol 2017; 69 (9): 1204-1205.

19. Viitasalo A, Lakka TA, Laaksonen DE et al. Validation of metabolic syndrome score by confirmatory factor analysis in children and adults and prediction of cardiometabolic outcomes in adults. Diabetologia 2014; 57 (5): 940-949.

20. Gurka MJ, Golden SH, Musani SK et al. Independent associations between a metabolic syndrome severity score and future diabetes by sex and race: the Atherosclerosis Risk in Communities Study and Jackson Heart Study. Diabetologia 2017; 60 (7): 1261-1270.

21. Jung KJ, Jee YH, Jee SH. Metabolic Risk Score and Vascular Mortality Among Korean Adults. Asia Pac J Public Health 2017; 29 (2): $122-131$.

Received June 12, 2018. Accepted August 17, 2018. 